\title{
Chinese herbal medicine Xinji pill protects the heart from ischemia/reperfusion injury through the Akt/Nrf2 pathway
}

\author{
QIUZHEN YUAN $^{1 *}$, RUIMING CHEN ${ }^{2 *}$, XU ZHENG ${ }^{3 *}$, MAIXIA MENG $^{2 *}$, YUPING KAO $^{1}$, \\ JUNFENG LIU ${ }^{1}$, XUEFENG GAN ${ }^{1}$, MINJUAN SHI ${ }^{1}$, JUNMING FU ${ }^{1}$, SHANSHAN JIANG ${ }^{4}$ and HUIYAO YU ${ }^{1,2}$ \\ ${ }^{1}$ Department of Pharmacy, Shanxi Hospital of Traditional Chinese Medicine, Xi'an, Shaanxi 710032; \\ ${ }^{2}$ Institute of TCM Research, Shaanxi Provincial Academy of Traditional Chinese Medicine, Xianyang, Shaanxi 712046; \\ ${ }^{3}$ Department of Pharmacology, Shaanxi Institute for Food and Drug Control, Xi'an, Shaanxi 710000; \\ ${ }^{4}$ Scientific Research Department, Shaanxi Hospital of Traditional Chinese Medicine, Xi'an, Shaanxi 710032, P.R. China
}

Received March 22, 2016; Accepted March 6, 2017

DOI: $10.3892 / \mathrm{mmr} .2017 .6732$

\begin{abstract}
The cardioprotective drugs used for treatment against ischemia/reperfusion (MI/R) injury have been well evaluated and are considered inadequate. The Chinese herbal medicine formula, Xinji pill (XJP) has been used traditionally for the prevention and treatment of ischemic heart diseases for decades. In the present study, the cardioprotective effects of XJP against MI/R injury were assessed in vivo and its possible mechanism was examined. Male Sprague-Dawley rats were selected for establishing an MI/R model, which was induced by ischemia for $30 \mathrm{~min}$ followed by $24 \mathrm{~h}$ reperfusion. Drugs and saline were administered intragastrically from day 14 prior to MI/R. Blood samples were collected for biochemical detection. The rats were then sacrificed and cardiac muscle tissues were harvested. The mRNA expression levels of antioxidant genes were measured by reverse transcription-quantitative polymerase chain reaction and the protein levels were measured by western blotting. Pretreatment with XJP for 14 days protected the heart against I/R-induced myocardial function disorder, protected against heart injury, as demonstrated by normalized serum levels of lactate dehydrogenase and creatine kinase, and suppressed oxidative stress. XJP markedly upregulated the expression of antioxidant genes, including superoxide dismutase, catalase, glutathione reductase and glutathione peroxidase, and promoted the protein expression of heme oxygenase- 1 and NFE2-related factor 2 (Nrf2) in the heart tissues. Furthermore, Akt kinase was confirmed to be upstream of Nrf2 in the XJP
\end{abstract}

Correspondence to: Professor Huiyao Yu, Department of Pharmacy, Shanxi Hospital of Traditional Chinese Medicine, 4 Xihua Gate, Lianhu, Xi'an, Shaanxi 710032, P.R. China

E-mail: yuhy5806@126.com

${ }^{*}$ Contributed equally

Key words: Xinji pill, oxidative stress, myocardial ischemia/reperfusion, Akt, NFE2-related factor 2 treatment. LY294002, a specific inhibitor of Akt, significantly eliminated the cardioprotective effects of XJP. In conclusion, these results demonstrated that XJP exhibited notable cardioprotective properties, in which the Akt/Nrf2 signaling pathway may be involved.

\section{Introduction}

Heart ischemia, with high morbidity and mortality rates, is a public health concern worldwide. To reduce ischemic myocardial injury and infarct size, the most effective therapy is efficient myocardial reperfusion. However, this process can induce further myocardial injury, known as myocardial ischemia/reperfusion (MI/R) injury $(1,2)$. MI/R injury was first described by Jennings et al in 1960. It was observed that reperfusion accelerated the development of necrosis in a canine coronary ligation model and histological changes were observed following I/R (3). I/R injury comprises distinct phases of cellular injury. During ischemia, ATP depletion, lactate accumulation and acidosis are observed, and during reperfusion, the production of reactive oxygen and nitrogen species are observed (4). Every year, $1,000,000$ individuals suffer from myocardial infarction in the United States alone, and almost $50 \%$ of cases of myocardial infarction occur following $I / R$ (5). Therefore, protecting the heart from MI/R injury is a primary goal of therapeutic intervention.

It is now well established that oxidative stress resulting from $\mathrm{I} / \mathrm{R}$ is important in the initiation and development of heart failure (6) Upon reperfusion of heart ischemic tissue, rebound hyperoxia and the oxidation of reduced intermediates causes a burst of reactive oxygen species (ROS) generation (7). In this acute phase, primary sources of ROS are predominantly from the mitochondrial respiratory chain and xanthine oxidase reaction. Cytokines released from damaged cells due to the inflammatory response also cause delayed and amplified generation of ROS (8). The protective effect of antioxidants has provided indirect evidence for a role of oxyradicals in I/R-induced myocardial damage (9). This evidences suggests that antioxidant therapies may be effective in protecting against $\mathrm{I} / \mathrm{R}$ injury and that the administration of antioxidants may provide potential benefit by attenuating 
oxidative stress $(10,11)$. Therefore, the screening of antioxidant agents in natural products to mitigate oxidative stress is necessary.

The transcription factor, NFE2-related factor 2 (Nrf2), is a member of the cap 'n' collar family and is a master regulator of cellular redox status (12). In oxidative stress, $\mathrm{Nrf} 2$ is free from kelch-like ECH-associated protein 1, a rapid ubiquitination causing the degradation of $\mathrm{Nrf} 2$, and then translocates from the cytoplasm into the nucleus. It upregulates the expression of numerous cytoprotective phase II detoxifying enzymes and antioxidant genes, which together suppress oxidative stress in the heart, and serve as a negative regulator of maladaptive cardiac remodeling and dysfunction (13). Based on the above, $\mathrm{Nrf2}$ is important in maintaining the functional integrity of the heart under oxidative stress.

As the active ingredients of traditional Chinese medicine (TCM) have multiple targets, there has been increasing interest in the treatment of MI/R injury with TCM (14). Among these, Xinji pill (XJP), a compound of Chinese medicine, is a good example. It was approved by the China State Food and Drug Administration in 1997 and is used for the treatment of angina pectoris and cardiac dysfunction. XJP has been commonly used clinically for the integrative treatment of patients with viral or ischemic heart disease, and has been well evaluated in the patients (15). The active components of XJP are extracted from Codonopsis pilosula, Herba epimedii, Salvia miltiorrhiza, Carthamus tinctorius, Radix puerariae, Fructus trichosanthis, Allium macrostemon and Coptis chinensis. However, the therapeutic mechanism of XJP against MI/R injury remains to be fully elucidated. The present study aimed to verify the cardioprotective effect of XJP during MI/R and elucidate the potential mechanisms.

\section{Materials and methods}

Experimental animals. Adult male Sprague-Dawley rats $(\mathrm{n}=60,250 \pm 15 \mathrm{~g}$ body weight) were obtained from the Animal Experimental Center of the Fourth Military Medical University (Xi'an, China). The protocols of the experiments were approved by the Ethics Committee for Animal Experimentation, and performed according to the Revised Guidelines for Animal Experimentation of the Fourth Military Medical University and the National Institute of Health Guide for the Care and Use of Laboratory Animals published by the National Academy of Sciences (8th edition, Washington DC, 2011) (16). The rats were fed in an environment with a 12 -h light-dark cycle at $25^{\circ} \mathrm{C}$.

Drug and reagents. XJP (batch no. 20151108) was obtained from Shaanxi Hospital of Traditional Chinese Medicine (Xi'an, China), which was prepared from water and ethanol extracts of Codonopsis pilosula, Herba epimedii, Salvia miltiorrhiza, Carthamus tinctorius, Radix puerariae, Fructus trichosanthis, Allium macrostemon and Coptis chinensis according to the guidelines of Good Manufacturing Practice and Good Laboratory Practice (17,18). The Hospital Agency and Shaanxi Provincial Food and Drug Administration (Xi'an, China) determined the content of its major components. Saline was used to dissolve XJP and produce solutions at concentrations of 20,40 and $80 \mathrm{mg} / \mathrm{ml}$ for experiments.
Chloral hydrate was purchased from Tianjin Kermel Chemical Reagent Co., Ltd. (Tianjin, China), which was freshly prepared prior to experiments in a 5\% solution with saline. Kits for the BCA protein assessment and detection of malondialdehyde (MDA) were purchased from Nanjing Jiancheng Bioengineering Institute (Nanjing, China). The antibodies against $\mathrm{Nrf2}$ (cat. no. sc-13032), heme oxygenase-1 (HO-1; cat. no. sc-10789), Akt (cat. no. sc-8312) and phosphorylated (p) Akt (cat. no. sc-33437) were obtained from Santa Cruz Biotechnology, Inc. (Santa Cruz, CA, USA). 2',7'-dichlorofluorescein diacetate (DCFH-DA) was purchased from Molecular Probes; Thermo Fisher Scientific, Inc. (Waltham, MA, USA).

Rat MI/R injury model and drug administration. The rats were randomly divided into the following groups: Sham, $\mathrm{I} / \mathrm{R}, \mathrm{XJP} 20 \mathrm{mg} / \mathrm{ml}+\mathrm{I} / \mathrm{R}$, XJP $40 \mathrm{mg} / \mathrm{ml}+\mathrm{I} / \mathrm{R}$ and XJP $80 \mathrm{mg} / \mathrm{ml}+\mathrm{I} / \mathrm{R}$. There were 6 rats in each group. The rats in the treatment groups were intragastrically administered with XJP in saline once daily for 14 days prior to I/R surgery. The rats in the Sham group and I/R groups received saline solution alone at the same time.

The rats were anesthetized by intraperitoneal injection by $5 \%$ chloral hydrate at a dose of $350 \mathrm{mg} / \mathrm{kg}$ and all experimental animal body temperatures were maintained at $37^{\circ} \mathrm{C}$. Following performing of left thoracotomy and exposure of the hearts, a 6-0 silk suture was passed underneath the LAD (2-3 mm inferior to the left auricle) and a slipknot was tied. $\mathrm{MI} / \mathrm{R}$ was induced by $30 \mathrm{~min}$ of ischemia followed by $24 \mathrm{~h}$ of reperfusion. Significant changes, including widening of the QRS complex and elevation of the ST segment detected using electroencephalography, were indicators of successful coronary occlusion. The animals in the Sham group underwent the same procedure but without ligation with suture silk. Blood was collected from the abdominal aorta $24 \mathrm{~h}$ following reperfusion, and the hearts were immediately removed and rinsed with pre-cooled saline, prior to being rapidly frozen in liquid nitrogen and preserved at $-80^{\circ} \mathrm{C}$.

Cardiac function and myocardial infarct size determination. At $24 \mathrm{~h}$ post-reperfusion, cannulation, which was connected to a biofunction experiment system (MP100-CE; Biopac Systems, Inc., Santa Barbara, CA, USA), was performed to the left ventricle through the right carotid artery following induction of anesthesia with 5\% chloral hydrate by intraperitoneal injection. Heart rate (HR), left ventricular systolic pressure (LVSP), and left ventricular maximum upstroke and descent velocity (+dp/dt $\max /-\mathrm{dp} / \mathrm{dt} \max$ ) were measured by computer algorithms and an interactive videographics program (Po-Ne-Mah Physiology Platform P3 Plus; Gould Instrument Systems, Valley View, OH, USA). The MI size was determined using the Evans blue/TTC double staining method as described previously (19) following completion of functional determination.

Lactate dehydrogenase $(\mathrm{LDH})$ and creatinine kinase $(\mathrm{CK})$ release evaluation. To isolate serum, blood samples were collected from the abdominal aorta at $24 \mathrm{~h}$ post-reperfusion and centrifuged at $3,250 \mathrm{xg}$ for $10 \mathrm{~min}$ at $4^{\circ} \mathrm{C}$. The activities of $\mathrm{LDH}$ and $\mathrm{CK}$ in plasma were measured to evaluate myocardial damage using commercially available assay kits (Nanjing Jiancheng Bioengineering Institute, Nanjing, China). 
ROS levels in the myocardial mitochondria. A DCFH-DA fluorescent probe detection kit was used to measure the intramitochondrial ROS levels. Mitochondria in myocardial tissue were extracted and purified by a tissue mitochondria isolation kit from Beyotime (Beyotime Institute of Biotechnology, Haimen, China) Purified mitochondria were incubated with DCFH-DA $(1 \mu \mathrm{M})$ at $37^{\circ} \mathrm{C}$ for $30 \mathrm{~min}$ and then washed three times with phosphate-buffered saline. The DCFH-DA-loaded mitochondria were excited at $488 \mathrm{~nm}$, and the fluorescence emission was collected at $525 \mathrm{~nm}$.

Tissue MDA content analysis. For the determination of MDA content, cardiac tissues were collected as described above and measured using a commercial kit from Nanjing Jiancheng Bioengineering Institute. Briefly, tissue homogenate was added to phosphate-buffered saline solution and ultra-sonication was used for complete homogenization. Following centrifugation at $1,500 \mathrm{x} \mathrm{g}$ at $4^{\circ} \mathrm{C}$ for $15 \mathrm{~min}$, the supernatants were collected into glass tubes. The supernatants were reacted with sodium acetate solution containing thiobarbituric acid at $95^{\circ} \mathrm{C}$ for $40 \mathrm{~min}$. Following centrifugation at $1,500 \mathrm{x} \mathrm{g}$ for $15 \mathrm{~min}$ at room temperature, the supernatants were collected and the reaction products were measured spectrophotometrically at $532 \mathrm{~nm}$ absorbance and expressed as MDA equivalents (pmol) per milligram of proteins.

Western blot analysis. Following reperfusion, 50 mg of myocardial tissue was collected and stored at $-80^{\circ} \mathrm{C}$. Radioimmunoprecipitation assay lysis buffer was used to extract the whole protein, and the concentration was determined using a BCA protein kit according to the manufacturer's protocol instructions, with the mean concentration values computed. The assessment was performed twice for each sample, and the values were averaged. The samples were stored at $-80^{\circ} \mathrm{C}$.

Equal quantities $(30 \mu \mathrm{g})$ of protein was separated on a $10 \%$ Tris-glycine SDS-PAGE polyacrylamide gel and transferred onto polyvinylidene fluoride membranes (Invitrogen; Thermo Fisher Scientific, Inc.). Following blocking for $1.5 \mathrm{~h}$ with a $5 \%$ solution of skim milk, membranes were incubated with primary antibodies against $\mathrm{Nrf} 2, \mathrm{HO}-1, \mathrm{Akt}, \mathrm{p}-\mathrm{Akt}$ and GAPDH at 1:1,000 dilution at $4{ }^{\circ} \mathrm{C}$ overnight, followed by the horseradish peroxidase-linked anti-goat antibody for $60 \mathrm{~min}$ at $37^{\circ} \mathrm{C}$. Following washing with Tris-buffered saline, the relative intensity of protein signals were normalized to the corresponding GAPDH intensity and quantified using densitometric analysis with QuantityOne software version 4.62 (Bio-Rad Laboratories, Inc., Hercules, CA, USA).

Reverse transcription-quantitative polymerase chain reaction (RT-qPCR) analysis. TRIzol lysis buffer was used to extract total RNA and concentrations were measured using a ND-1000 spectrophotometer (Nano-Drop Technologies; Thermo Fisher Scientific, Inc.). cDNA was synthesized, according to the manufacturer's protocol. The mRNA expression levels of superoxide dismutase (SOD), glutathione reductase (GSR), catalase (CAT) and glutathione peroxidase (GSH-Px) were determined using RT-qPCR analysis in a Step One Real-Time PCR system (Applied Biosystems; Thermo Fisher Scientific, Inc.). For each sample, a $14 \mu \mathrm{l}$ mix containing $1 \mu \mathrm{g}$ total RNA, $1 \mu \mathrm{l}$ of a $50 \mu \mathrm{M}$ Oligo (dT), $1 \mu \mathrm{l}$ of a $10 \mathrm{mM}$ dNTP mix and
RNase-free $\mathrm{dH}_{2} \mathrm{O}$ was heated at $65^{\circ} \mathrm{C}$ for $5 \mathrm{~min}$, and cooled on ice. After this, a $6 \mu 1$ mixture containing $1 \mu 1200 \mathrm{U} / \mu 1$ SuperScript $^{\mathrm{TM}}$ III Reverse Transcriptase, $4 \mu 1$ 5X First-Strand Buffer (Invitrogen; Thermo Fisher Scientific, Inc.) and $1 \mu \mathrm{l}$ of $40 \mathrm{U} / \mu 1$ RNase inhibitor was added. Subsequently, reverse transcription was performed with an initial priming step at $30^{\circ} \mathrm{C}$ for $10 \mathrm{~min}$, followed by cDNA synthesis at $42^{\circ} \mathrm{C}$ for $50 \mathrm{~min}$. Activation was stopped at $70^{\circ} \mathrm{C}$ for $15 \mathrm{~min}$. RT-qPCR was performed with SYBR-Green-based detection reagents (Roche Applied Science, Penzberg, Germany). Thermal cycling conditions were as follows: An initial predenaturation step at $94^{\circ} \mathrm{C}$ for $10 \mathrm{~min}$, followed by 35 cycles of denaturation at $94^{\circ} \mathrm{C}$ for $1 \mathrm{~min}$, annealing at $58^{\circ} \mathrm{C}$ for $50 \mathrm{sec}$, extension at $72^{\circ} \mathrm{C}$ for $1 \mathrm{~min}$ and a final extension step at $72^{\circ} \mathrm{C}$ for $7 \mathrm{~min}$. Serial dilutions of cDNA from the Sham surgery group were used to produce standard curves. cDNA was run in duplicate. To account for differences in cDNA preparation and cDNA amplification efficiency, the mRNA expression was normalized to that of GAPDH. To reliably estimate changes in mRNA levels, a standard curve for each mRNA was generated using serial dilutions of a standard cDNA, as described previously (20). The relative data for each gene were calculated by the $2^{-\Delta \Delta \mathrm{Cq}}$ method (21). A value of 1 was attributed to sham and all mRNA levels were expressed as an $n$-fold difference relative to the sham. The sequences of the primers used were as follows: SOD sense, 5'AGATGACTT GGGCAAAGGTG3' and antisense, 5'CAATCCCAATCACAC CACAA3'; CAT sense, 5'ACATGGTCTGGGACTTCTGG3' and antisense, 5'CCATTCGCATTAACCAGCTT3'; GAPDH sense, 5'CCATCACTGCCACTCAGAAGAC3' and antisense, 5'TCATTGGCAGGTTTCTCCA3'; GSH-Px sense, 5'GAT TCGTTCCAAACTTCCTGCTA3' and antisense, 5'GCTCCC AGAACAGCCTGTTG3'; GSR sense, 5'GGAAGTCAACGG GAAGAAGTTCACTG3' and antisense, 5'CAATGTAACCGG CACCCACAATAAC3'.

Caspase-3 activity. A fluorometric assay kit was used to measure caspase-3 activity according to the manufacturer's protocol. In brief, heart tissues were lysed in $50 \mu \mathrm{l}$ of ice-cold lysis buffer at $4^{\circ} \mathrm{C}$ for $30 \mathrm{~min}$, and then centrifuged at $12,000 \mathrm{~g}$ at $4^{\circ} \mathrm{C}$ for $10 \mathrm{~min}$. A BCA protein assay kit was used to quantify the protein concentrations in the supernatants. Reaction buffer $(50 \mu \mathrm{l})$ and caspase-3 substrate $(5 \mu \mathrm{l})$ were added to the lysates (40 $\mu \mathrm{g}$ per assay). Following incubation for $4 \mathrm{~h}$ at $37^{\circ} \mathrm{C}$, the fluorescence was measured using a microplate reader with excitation at $400 \mathrm{~nm}$ (Fluoroskan Ascent; Thermo Labsystems, Santa Rosa, CA, USA).

Statistical analysis. All values are expressed as the mean \pm standard deviation and analyzed using SPSS 18.0 statistical software (SPSS, Inc., Chicago, IL, USA. The difference between two groups was analyzed using one-way analysis of variance followed by Tukey's test for multiple comparisons. $\mathrm{P}<0.05$ was considered to indicate a statistically significant difference.

\section{Results}

XJP ameliorates myocardial function. The effect of XJP on LVSP and the $\pm \mathrm{dp} / \mathrm{dt}$ max of the left ventricle following $\mathrm{MI} / \mathrm{R}$ for $24 \mathrm{~h}$ were evaluated. The rats treated with XJP had 

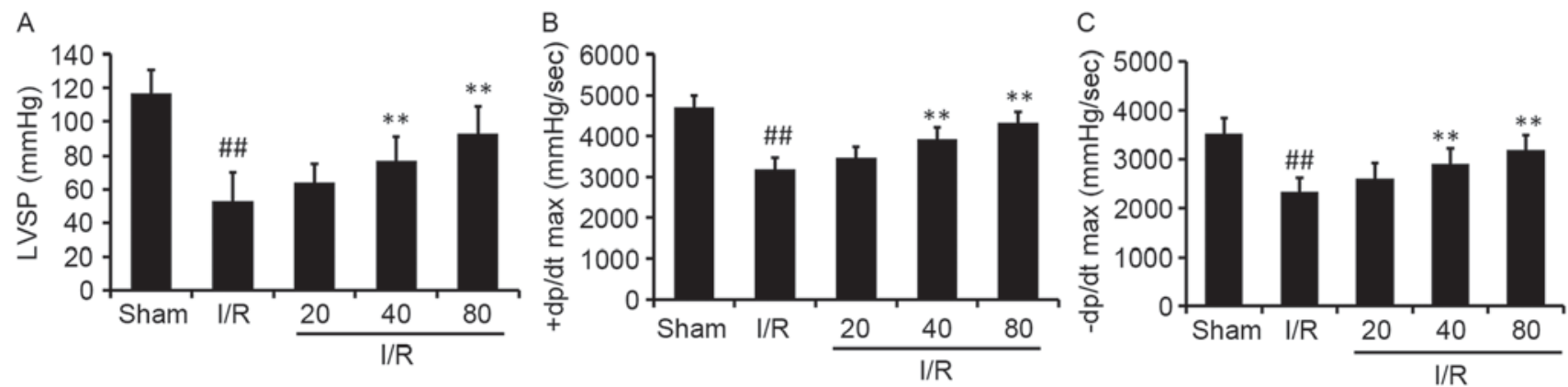

Figure 1. Hemodynamic assessment in rats following I/R-induced injury. Quantitative assessment of hemodynamics on cardiac function based on (A) LVSP, (B) $+\mathrm{dp} / \mathrm{dt} \max$ and (C) -dp/dt max. Data are expressed as the mean \pm standard deviation from six animals. ${ }^{\# \#} \mathrm{P}<0.01$, vs Sham group; ${ }^{* *} \mathrm{P}<0.01$, vs. I/R group. LVSP, left ventricular systolic pressure; +dp/dt max, left ventricular maximum upstroke velocity; -dp/dt max, left ventricular maximum descent velocity; I/R, ischemia/reperfusion.
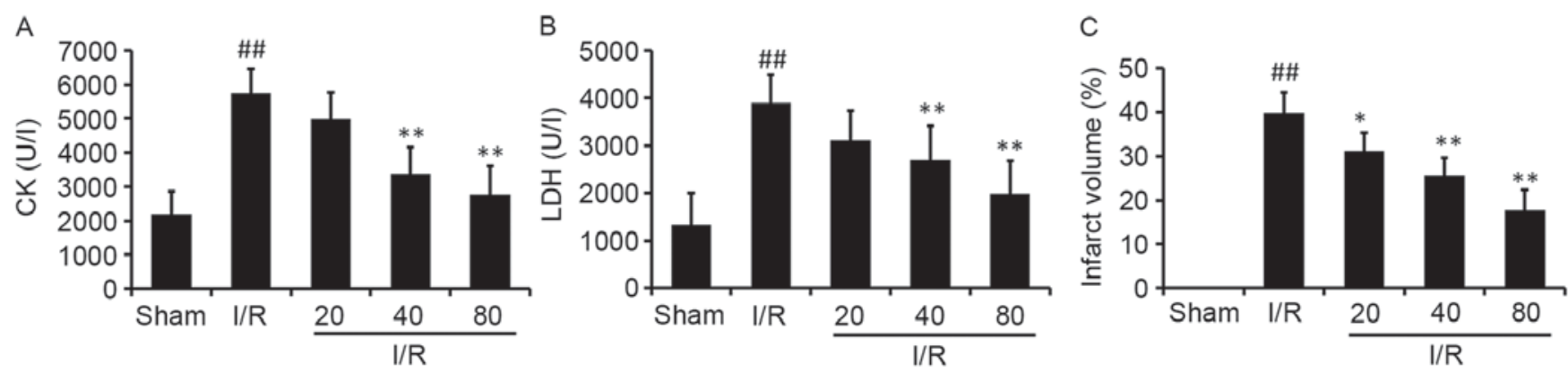

Figure 2. Effect of XJP on the content of CK and LDH in rat serum and changes in infarct volume. Statistical results from ELISA for (A) CK and (B) LDH Pretreatment with XJP for 14 days prior to I/R (30 min/24 h). (C) Infarct size was determined using the Evans blue/TTC double staining method. Data are expressed as the mean \pm standard deviation ( $\mathrm{n}=8$ /group). ${ }^{\#} \mathrm{P}<0.01$, vs. Sham group; ${ }^{*} \mathrm{P}<0.05$ and ${ }^{* *} \mathrm{P}<0.01$, vs. I/R group. XJP, Xinji pill; CK, creatine kinase; LDH, lactate dehydrogenase; I/R, ischemia/reperfusion.

significantly improved LVSP, +dp/dt max and -dp/dt max $24 \mathrm{~h}$ following I/R, compared with I/R-treated animals (Fig. 1). The $\mathrm{HR}$ and mean arterial blood pressure (MABP) among groups were also evaluated, with no significant differences in the changes. These results suggested that XJP treatment provided benefits for myocardial function recovery following MI/R.

$X J P$ reduces $M I / R$ injury. To determine the cardioprotective effects of XJP pretreatment on the injury induced by $I / R$, cardiac enzyme levels (CK and LDH) were determined using a microplate reader. The results showed that the rats in the model group had significantly higher levels of CK and LDH, compared with those in the Sham group (Fig. 2A and B). Compared with the I/R group, the XJP treatment groups had significantly decreased levels of CK and LDH in a dose-dependent manner. This indicated that XJP may have protected the cardiomyocytes from injury following $\mathrm{MI} / \mathrm{R}$.

The effect of XJP on infarct size was also examined (Fig. 2C). It was found that the infarct size was significantly increased by I/R treatment, compared with the sham group, and the size was significantly reduced by XJP treatment, compared with that in the $\mathrm{I} / \mathrm{R}$ group. The results revealed for infarct size and cardiac enzymes suggested that XJP was useful in attenuating MI/R injury.

$X J P$ reduces myocardial ROS and MDA levels. Acute I/R can induce the enhanced generation of high ROS; therefore, cardiac levels of ROS and MDA were measured. As shown in Fig. 3,
MDA levels in the I/R-treated rats were significantly higher, compared with those in the Sham rats $(\mathrm{P}<0.01)$. Pretreatment of the rats with XJP at concentrations of 40 or $80 \mathrm{mg} / \mathrm{kg}$ significantly decreased MDA levels, compared with those in the model group (Fig. 3A). In addition, in the I/R group, ROS levels were higher, compared with those in the Sham group in myocardial mitochondria, as shown in the fluorescence intensity assay, however, in the rats pretreated with XJP, ROS levels were significantly decreased, compared with those in the I/R group (Fig. 3B).

XJP increases the mRNA expression of antioxidant proteins. At the end of reperfusion, the mRNA levels of SOD, GSR, CAT and GSH-Px were quantified using RT-qPCR analysis (Fig. 4). In the I/R group, the mRNA levels of SOD, GSR, CAT and GSH-Px were significantly decreased $(\mathrm{P}<0.01)$, compared with those in the Sham group, whereas the mRNA expression of the antioxidant proteins in the XJP-treated groups were significantly higher, compared with those in the $\mathrm{I} / \mathrm{R}$ group $(\mathrm{P}<0.01)$.

$X J P$ increases the phosphorylation of Akt and the expression of $N r f 2$. Nrf2, a crucial regulator against oxidative stress, can regulate the expression of antioxidant proteins. Therefore, the present study examined the effects of XJP on the Nrf2 signaling pathway. HO-1, a cytoprotective downstream target protein of $\mathrm{Nrf} 2$, is a typical antioxidant. To determine the potential effects of XJP on the Nrf2 signaling pathway in vivo, the expression of $\mathrm{Nrf} 2$ and $\mathrm{HO}-1$ were analyzed. As shown 
A

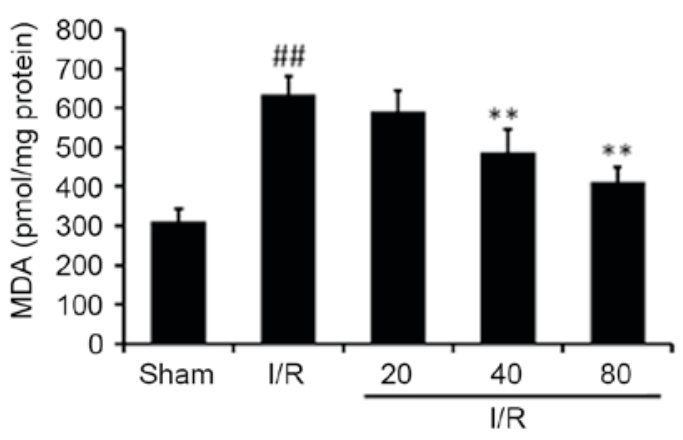

B

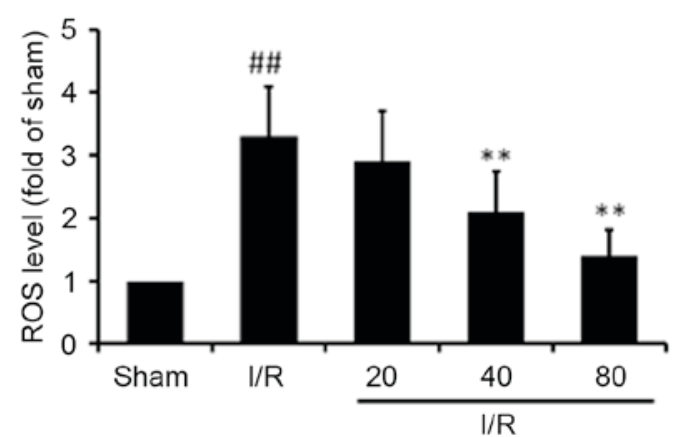

Figure 3. Effect of XJP on the content of MDA and ROS in rats following I/R-induced injury. (A) MDA was quantitative assessed using an ELISA kit according to the manufacturer's protocol. (B) A 2',7'-dichlorofluorescein diacetate fluorescent probe detection kit was to detect the levels of ROS in myocardial mitochondria. Data are expressed as the mean \pm standard deviation ( $\mathrm{n}=6 /$ group). ${ }^{\# \#} \mathrm{P}<0.01$, vs. Sham group; ${ }^{* *} \mathrm{P}<0.01$, vs. I/R group. XJP, Xinji pill; MDA, malondialdehyde; ROS, reactive oxygen species; I/R, ischemia/reperfusion.
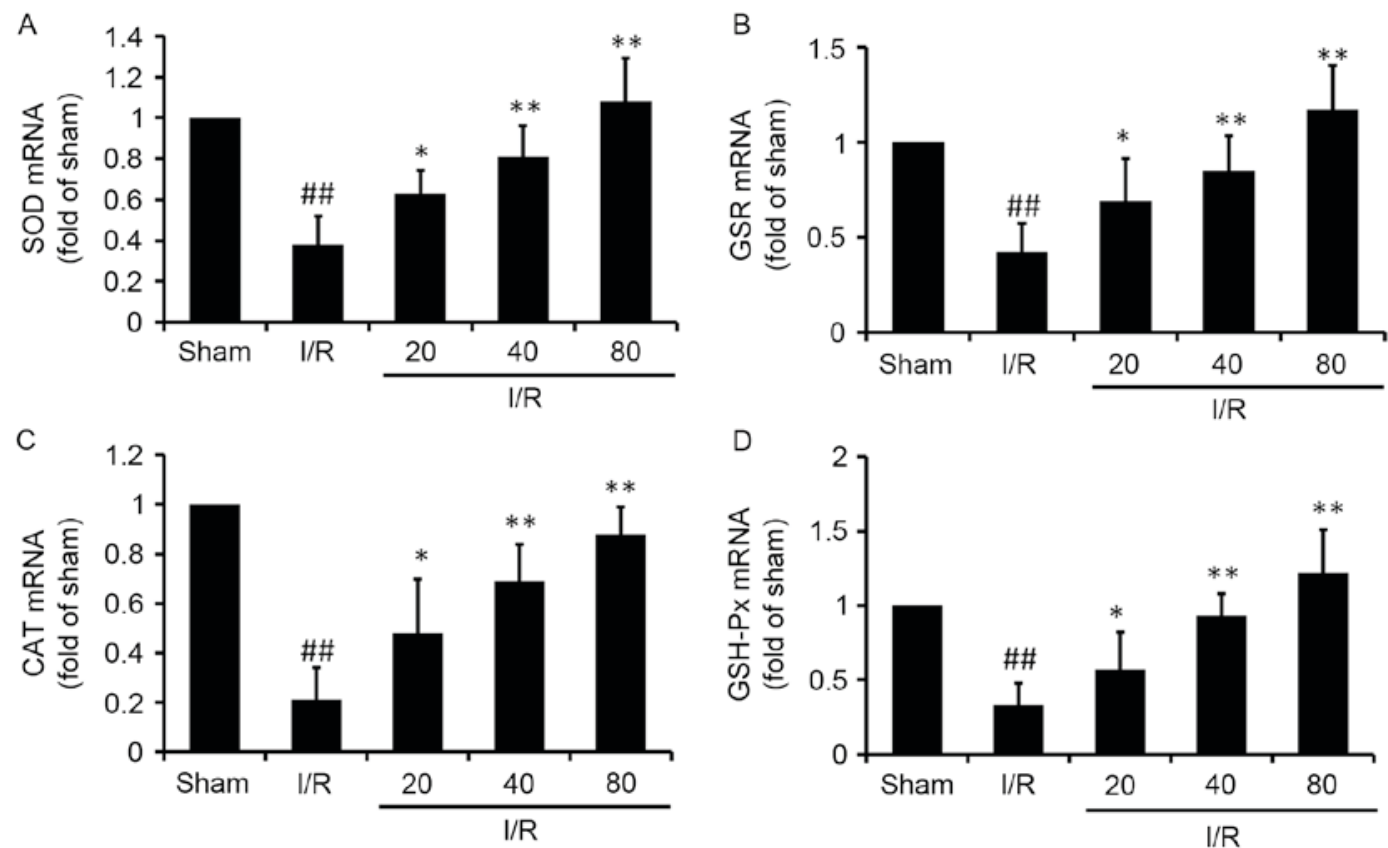

Figure 4. XJP regulates the mRNA expression of SOD, GSR, CAT and GSH-Px in rat hearts subjected to I/R. Reverse transcription-quantitative polymerase chain reaction analysis was used to assess the relative mRNA levels of cardiac (A) SOD, (B) GSR, (C) CAT and (D) GSH-Px. Results were normalized to GAPDH. Data are expressed as the mean \pm standard deviation ( $\mathrm{n}=6$ /group). ${ }^{\# \#} \mathrm{P}<0.01$, vs. Sham group; ${ }^{*} \mathrm{P}<0.05$ and ${ }^{* *} \mathrm{P}<0.01$, vs. I/R group. XJP, Xinji pill; I/R, ischemia/reperfusion; SOD, superoxide dismutase; GSR, glutathione reductase; CAT, catalase; GSH-Px, glutathione peroxidase.

in Fig. 5, I/R induced a marginal increase in the expression levels of Nrf2 and HO-1, compared with those in the Sham group. The expression levels of Nrf2 and HO-1 were further increased by XJP pretreatment in a dose-dependent manner.

Akt pathway mediates the activation of Nrf2 by XJP. It has been shown that activation of the Akt pathway can protect the heart from I/R injury (22). To further investigate upstream of Nrf2 and the mechanisms underlying the XJP-induced cardioprotective effects, the activation of Akt was measured using western blot analysis for rats subjected to MI/R (Fig. 6A). The results showed that the phosphorylation of Akt in the heart was significantly decreased by $\mathrm{I} / \mathrm{R}$ treatment, compared with that in the sham group $(\mathrm{P}<0.01)$. However, pretreatment with XJP for 14 days significantly increased the phosphorylation of Akt in the heart. These results suggested that Akt kinase may have be involved in the XJP-stimulated activation of Nrf2.

To further confirm these findings, an inhibitor of phosphoinositide 3-kinase (PI3K)/Akt signaling, LY294002 was used subsequent experiments. As shown in Fig. 6B, the XJP-induced expression of Nrf2 and HO-1 were effectively inhibited in the presence of LY294002. It was also observed that LY294002 eliminated the cardioprotective effects of XJP, which was shown by the changes in the levels of LDH (Fig. 6C) and MDA (Fig. 6D).

\section{Discussion}

The present study is the first, to the best of our knowledge, to examine the cardioprotective effects of XJP against MI/R 


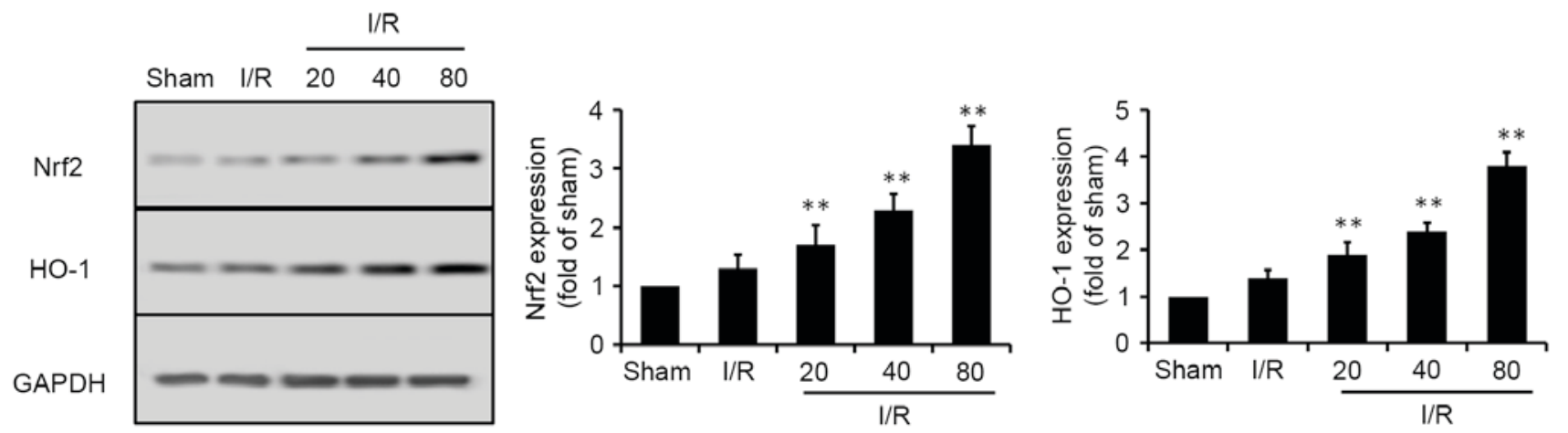

Figure 5. Effect of XJP on the expression of Nrf2 and HO-1 in heart tissues. Pretreatment with XJP for 14 days, followed by ischemia for 30 min and reperfusion for $24 \mathrm{~h}$. Heart tissues were collected for western blot analysis. Data are expressed as the mean \pm standard deviation ( $\mathrm{n}=6 /$ group). ${ }^{* *} \mathrm{P}<0.01$, vs. I/R group. XJP, Xinji pill; I/R, ischemia/reperfusion; Nfr2, NFE2-related factor 2; HO-1, heme oxygense-1.

A
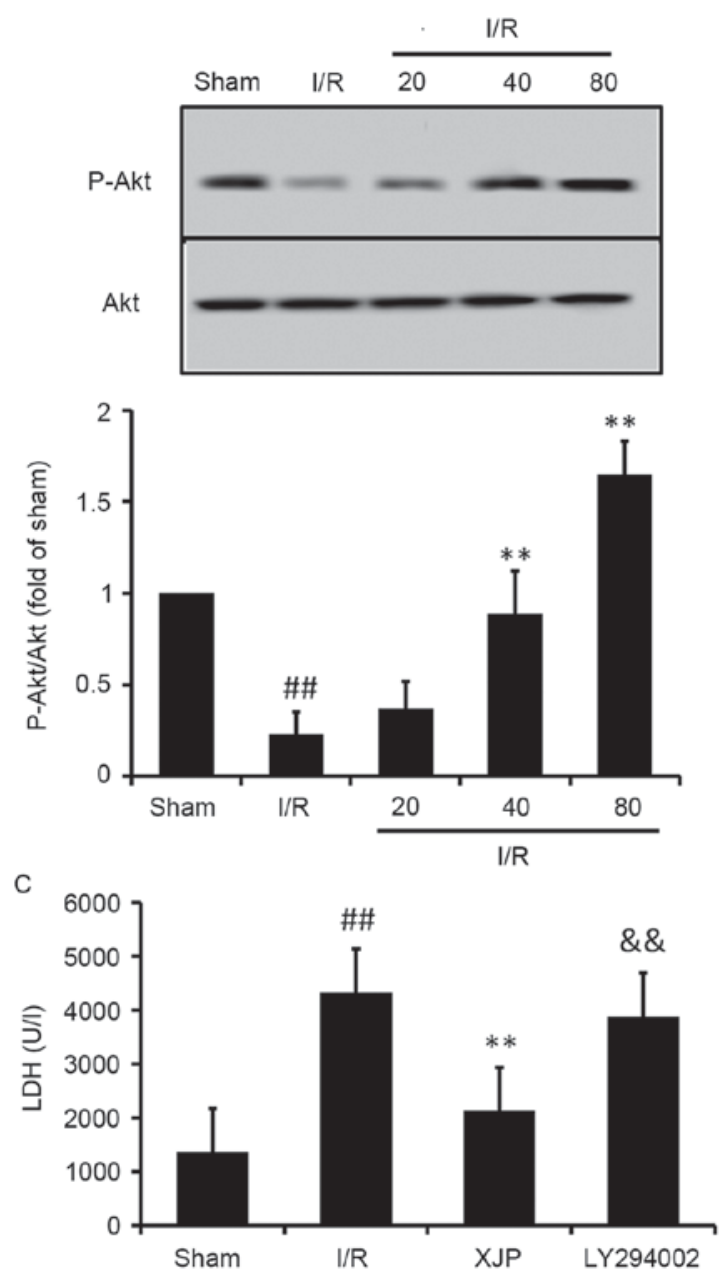
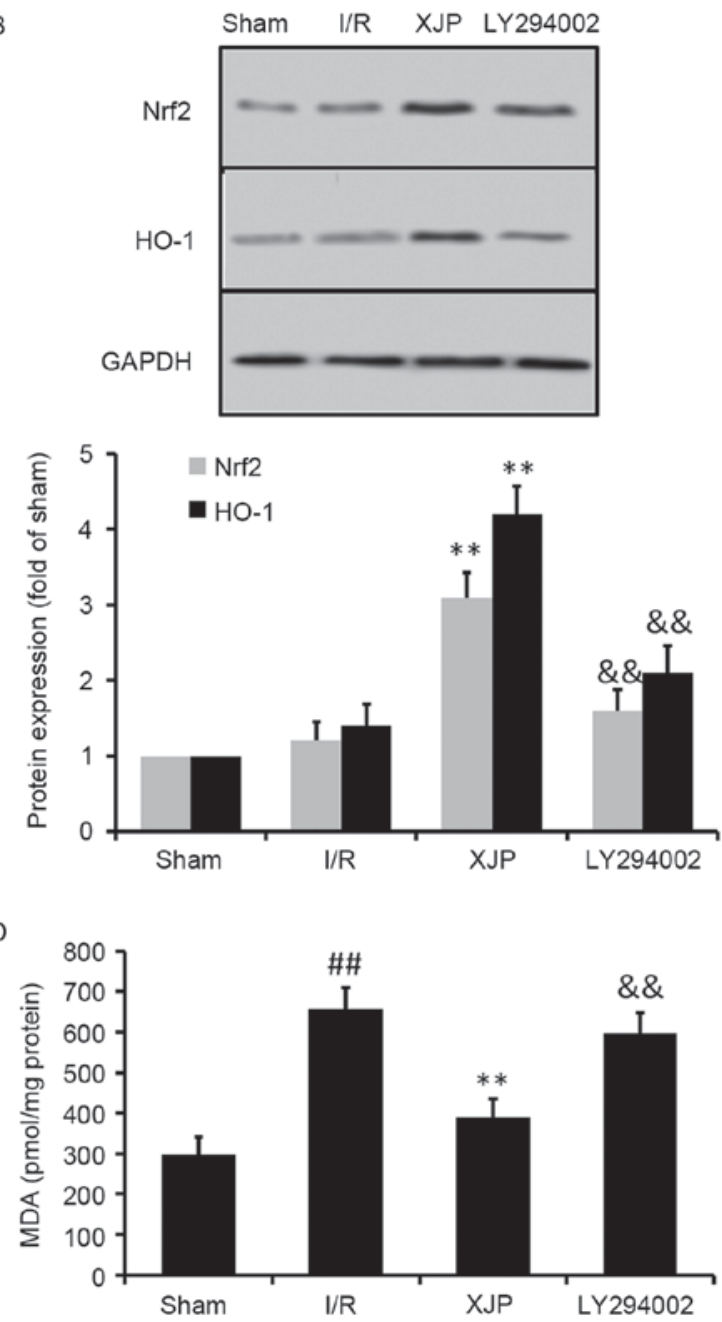

Figure 6. Role of Akt on the expression of Nrf2 by XJP in MI/R rats. (A) Phosphorylation of Akt induced by XJP. (B) Effects of inhibition of Akt in XJP-induced expression of Nrf2 and HO-1. Experiments aimed to determine the role of the Akt pathway in the protective effect of XJP against I/R-induced injury in rats, demonstrated by levels of (C) LDH and (D) MDA. Data are expressed as the mean \pm standard deviation (n=6/group). ${ }^{\# \#} \mathrm{P}<0.01$, vs. Sham group; ${ }^{* *} \mathrm{P}<0.01$, vs. I/R group; \&\& $\mathrm{P}<0.01$, vs. I/R+XJP group. XJP, Xinji pill; I/R, ischemia/reperfusion; Nfr2, NFE2-related factor 2; HO-1, heme oxygense-1; MDA, malondialdehyde; LDH, lactase dehydrogenase p-Akt, phosphorylated Akt.

injury in rats. It was found that pretreatment with XJP significantly improved cardiac function and reduced infarct size in the I/R rat hearts in vivo. These beneficial effects were demonstrated by the preservation of left ventricular function, as reflected by a significant increase in the indices of contraction
$(+\mathrm{dP} / \mathrm{dt} \max )$ and relaxation $(-\mathrm{dP} / \mathrm{dt} \max )$, and the increase in preload (LVSP). The results demonstrated that XJP reduced I/R-induced myocardial injury, increased the expression levels of Nrf2 and Akt, increased the levels of antioxidant proteins following MI/R, and inhibited necrosis in the $\mathrm{I} / \mathrm{R}$ myocardium. 
CK and LDH are used clinically as biomarkers of myocardial damage. They are expressed constitutively in endochylema and, in the normal physiological state, cannot transit through cytoplasmic membrane. $\mathrm{LDH}$ and $\mathrm{CK}$ are released from cells when the cell is damaged or dead, therefore, they are appropriate for the assessment of cellular injury (23-25). The activities of $\mathrm{LDH}$ and $\mathrm{CK}$ in the serum can represent the extent of myocardial injury induced by $\mathrm{I} / \mathrm{R}$. The results of the present study indicated that I/R significantly increased the serum levels of LDH and CK, whereas pretreatment with XJP significantly decreased these changes. These results showed that XJP had cardioprotective effects against I/R injury.

There are several reports that oxidative stress contributes to the pathogenesis of MI/R injury $(26,27)$. In normal physiological conditions, ROS are usually scavenged by antioxidants. In disease states, including sudden hypoxia, the overproduction of ROS and overconsumption of antioxidants results in oxidative cellular damage $(28,29)$. ROS can oxidize nucleic acids, proteins and lipids, and affect critical signal transduction pathways $(30,31)$. Finally ROS-induced abnormalities result in alterations of cardiac function, cardiac stunning, arrhythmias, cellular injury and death $(32,33)$. Cellular antioxidants can scavenge ROS and minimize injuries caused by the oxidative stress $(34,35)$. In the present study, it was found that I/R caused a rapid and significant increase in ROS generation and levels of MDA in the heart. Pretreatment with XJP eliminated these increases to a certain extent. These results suggested that the attenuation of oxidative stress was involved in the cardioprotective effect of XJP.

The removal of excess ROS requires several antioxidant enzymes. Among these, SOD can transform intracellular superoxide anions to $\mathrm{H}_{2} \mathrm{O}_{2}$, which can be scavenged by CAT and GSH-Px through enzymatic reactions. CAT, an enzyme located in the peroxisome, promotes the conversion of $\mathrm{H}_{2} \mathrm{O}_{2}$ to $\mathrm{H}_{2} \mathrm{O}$ and $\mathrm{O}_{2}$ (36). GSH-Px acts in conjunction with the GSH tripeptide, which is present in cells in high (micromolar) concentrations. GSH-Px decomposes peroxides to water and simultaneously oxidizes GSH (37). To further support the findings of the present study, the gene expression levels of SOD, GSR, CAT and GSH-Px were determined using RT-qPCR analysis. In the cardiac muscle tissue exposed to $I / R$, it was found that the expression levels of SOD, GSR, CAT and GSH-Px were significantly enhanced following treatment with XJP. Thus, the protective effect of XJP pretreatment may be achieved through upregulation in the gene expression levels of SOD, GSR, CAT and GSH-Px, and the subsequent inhibition of oxidative stress.

Nrf2, a redox-sensitive transcription factor, predominantly mediates the transcriptional regulation of antioxidant genes, including SOD, GSR, CAT and GSH-Px. It is expressed in multiple tissues, but is only activated in response to certain electrophilic agents and oxidative stress, including ROS, specific antioxidants and certain disease processes. Upon activation, the interaction between $\mathrm{Nrf} 2$ with the antioxidant-response element (ARE) mediates the induction of a series of cytoprotective proteins, including phase II enzymes SOD, GSH and CAT $(38,39)$. These findings suggest that activation of the Nrf2/ARE pathway may be involved in the gene expression and activities of SOD, CAT, GSH and GPx induced by XJP. In order to confirm this, the present study examined the effects of XJP on the protein expression levels of Nrf2 and HO-1. The data showed that XJP upregulated the expression of Nrf2 in the hearts subjected to I/R for the first time, demonstrating the activation of a cytoprotective pathway. Further investigation showed that XJP caused an increase in the expression of HO-1, which is a gene known to be upregulated by the activation of Nrf2.

A number of studies have identified that Akt is involved in the activation of Nrf2/ARE and its associated gene expression, and several studies have shown that several phytochemicals from herbal medicines, including butin and 3 a,4'-didemethylnobiletin, protect against oxidative stress-induced cell injury via the PI3K/Akt/Nrf2-dependent pathway (40). The present study investigated whether this pathway contributed to the protective effects of XJP against I/R-induced oxidative stress. Of note, the phosphorylation of Akt was significantly increased in the XJP-treated hearts in a dose-dependent manner, and the inhibition of Akt signaling by the Akt inhibitor, LY294002, completely inhibited the XJP-induced protein expression of Nrf2 and HO-1. Subsequent experiments showed that LY294002 eliminated the ability of XJP to control the levels of LDH and MDA, which were significantly increased by I/R. These results indicated that Akt/Nrf2 signaling was involved in the cytoprotective effects of XJP.

In conclusion, the present study demonstrated that XJP protected myocardial function and damage in rats exposed to $\mathrm{MI} / \mathrm{R}$ injury. It significantly decreased infarct volume, improved hemodynamics and alleviated myocardial damage. The cardioprotective effects of XJP against I/R injury may be attributed to the increasing activities and protein expression levels of certain antioxidative enzymes, including SOD, CAT, GSR and GSH-Px. These may occur through a mechanism involving the activation of Akt and the upregulted expression of Nrf2 and its downstream antioxidant genes. These findings provide insight into the protective potential of XJP in MI/R injury.

\section{Acknowledgements}

The present study was supported by the Key Project of Natural Science Foundation Research of Shaanxi province (grant no. 2014JZ2-006).

\section{References}

1. Zhong X, Li X, Qian L, Xu Y, Lu Y, Zhang J, Li N, Zhu X, Ben J, Yang Q and Chen Q: Glycine attenuates myocardial ischemia-reperfusion injury by inhibiting myocardial apoptosis in rats. J Biomed Res 26: 346-354, 2012.

2. Piper HM, García-Dorado D and Ovize M: A fresh look at reperfusion injury. Cardiovasc Res 38: 291-300, 1998.

3. Jennings RB, Sommers HM, Smyth GA, Flack HA and Linn H: Myocardial necrosis induced by temporary occlusion of a coronary artery in the dog. Arch Pathol 70: 68-78, 1960.

4. Armstrong SC: Protein kinase activation and myocardial ischemia/reperfusion injury. Cardiovasc Res 61: 427-436, 2004.

5. Go AS, Mozaffarian D, Roger VL, Benjamin EJ, Berry JD, Borden WB, Bravata DM, Dai S, Ford ES, Fox CS, et al: Heart disease and stroke statistics-2013 update: A report from the American Heart Association. Circulation 127: e6-e245, 2013.

6. Murphy E and Steenbergen C: Mechanisms underlying acute protection from cardiac ischemia-reperfusion injury. Physiol Rev 88: 581-609, 2008.

7. Becker LB: New concepts in reactive oxygen species and cardiovascular reperfusion physiology. Cardiovasc Res 61: 461-470, 2004. 
8. Frangogiannis NG, Smith CW and Entman ML: The inflammatory response in myocardial infarction. Cardiovasc Res 53: 31-47, 2002.

9. Laskowski A, Woodman OL, Cao AH, Drummond GR, Marshall T, Kaye DM and Ritchie RH: Antioxidant actions contribute to the antihypertrophic effects of atrial natriuretic peptide in neonatal rat cardiomyocytes. Cardiovas Resear 72: $112-123,2006$.

10. Moens AL, Claeys MJ, Timmermans JP and Vrints CJ: Myocardial ischemia/reperfusion-injury, a clinical view on a complex pathophysiological process. Int J Cardiol 100: 179-190, 2005 .

11. Rajak S, Banerjee SK, Sood S, Dinda AK, Gupta YK, Gupta SK and Maulik SK: Emblica officinalis causes myocardial adaptation and protects against oxidative stress in ischemic-reperfusion injury in rats. Phytother Res 18: 54-60, 2004.

12. Li J, Ichikawa T, Villacorta L, Janicki JS, Brower GL, Yamamoto $\mathrm{M}$ and Cui T: Nrf2 protects against maladaptive cardiac responses to hemodynamic stress. Arterioscler Thromb Vasc Biol 29: 1843-1850, 2009.

13. McMahon M, Itoh K, Yamamoto M and Hayes JD: Keap1dependent proteasomal degradation of transcription factor Nrf2 contributes to the negative regulation of antioxidant response element-driven gene expression. J Biol Chem 278: 21592-21600, 2003.

14. Boran AD and Iyengar R: Systems approaches to polypharmacology and drug discovery. Curr Opin Drug Discov Devel 13: 297-309, 2010

15. Zhang A and Hui A: Inhibition of coxsackie virus and effect on the treatment of viral myocarditis of Xinji Pill in mice. Shanx Tradit Chin Med 12: 563-564, 1998 (In Chinese).

16. National Research Council (US) Committee for the Update of the Guide for the Care and Use of Laboratory Animals: Guide for the Care and Use of Laboratory Animals, 8th edition. Nationa Academies Press (US), Washington, DC: pp1072-1073, 2011.

17. China's food and drug administration: Good laboratory practice. Standards Press of China, Beijing, pp54-59, 2003.

18. China's food and drug administration: Good manufacturing practice. Standards Press of China, Beijing, pp106-109, 2015.

19. Tao L, Gao E, Bryan NS, Qu Y, Liu HR, Hu A, Christopher TA Lopez BL, Yodoi J, Koch WJ, et al: Cardioprotective effects of thioredoxin in myocardial ischemia and reperfusion: Role of S-nitrosation (corrected). Proc Natl Acad Sci USA 101: 11471-11476, 2004

20. Cao Z and Li Y: Protecting against peroxynitrite-mediated cytotoxicity in vascular smooth muscle cells via upregulating endogenous glutathione biosynthesis by $3 \mathrm{H}$-1,2-dithiole-3-thione. Cardiovasc Toxicol 4: 339-353, 2004.

21. Livak KJ and Schmittgen TD: Analysis of relative gene expression data using real-time quantitative PCR and the 2(-Delta Delta C(T)) Method. Methods 25: 402-408, 2001.

22. Hu W, Zhang P, Gu J, Yu Q and Zhang D: NEDD4-1 protects against ischaemia/reperfusion-induced cardiomyocyte apoptosis via the PI3K/Akt pathway. Apoptosis 22: 437-448, 2017.

23. Cho MH, Niles A, Huang R, Inglese J, Austin CP, Riss T and Xia M: A bioluminescent cytotoxicity assay for assessment of membrane integrity using a proteolytic biomarker. Toxicol In Vitro 22: 1099-1106, 2008.
24. Corey MJ, Kinders RJ, Brown LG and Vessella RL: A very sensitive coupled luminescent assay for cytotoxicity and complement-mediated lysis. J Immunol Methods 207: 43-51, 1997.

25. Kim H, Yoon SC, Lee TY and Jeong D: Discriminative cytotoxicity assessment based on various cellular damages. Toxicol Lett 184: 13-17, 2009.

26. Wu Y and Yuan BX: Effects of Qiangxin capsules on myocardial reperfusion arrhythmias in rats. Northwest Pharmac J 16: 23-24, 2001.

27. Fu J, Huang H, Liu J, Pi R, Chen J and Liu P: Tanshinone IIA protects cardiac myocytes against oxidative stress-triggered damage and apoptosis. Eur J Pharmac 568: 213-221, 2007.

28. Halliwell B and Gutteridge JMC: Free radicals in biology and medicine, 3rd edition. Oxford, Clarendon Press, pp246-350, 1999.

29. Maxwell SR and Lip GY: Reperfusion injury: A review of the pathophysiology, clinical manifestations and therapeutic options. Int J Cardiol 58: 95-117, 1997.

30. Duranteau J, Chandel NS, Kulisz A, Shao Z and Schumacker PT: Intracellular signaling by reactive oxygen species during hypoxia in cardiomyocytes. J Biol Chem 273: 11619-11624, 1998.

31. Hensley K, Robinson KA, Gabbita SP, Salsman S and Floyd RA Reactive oxygen species, cell signaling, and cell injury. Free Radic Biol Med 28: 1456-1462, 2000.

32. Das DK and Maulik N: Antioxidant effectiveness in ischemia reperfusion tissue injury. Methods Enzymol 233: 601-610, 1994.

33. Lefer DJ and Granger DN: Oxidative stress and cardiac disease. Am J Med 109: 315-323, 2000.

34. Ferrari R, Ceconi C, Curello S, Cargnoni A, Alfieri O, Pardini A, Marzollo P and Visioli O: Oxygen free radicals and myocardial damage: Protective role of thiolcontaining agents. Am J Med 91 (Suppl): 95-105, 1991.

35. Frei B: On the role of vitamin $\mathrm{C}$ and other antioxidants in atherogenesis and vascular dysfunction. Proc Soc Exp Biol Med 222: 196-204, 1999

36. Valko M, Rhodes CJ, Moncol J, Izakovic M and Mazur M: Free radicals, metals and antioxidants in oxidative stress-induced cancer. Chem Biol Interact 160: 1-40, 2006.

37. Tengattini S, Reiter RJ, Tan DX, Terron MP, Rodella LF and Rezzani R: Cardiovascular diseases: Protective effects of melatonin. J Pineal Res 44: 16-25, 2008.

38. Ma Q, Kinneer K, Bi Y, Chan JY and Kan YW: Induction of murine NAD(P)H:quinine oxidoreductase by 2,3,7,8-tetrachlorodibenzo-p-dioxin requires the CNC (cap 'n' collar) basic leucine zipper transcription factor Nrf2 (nuclear factor erythroid 2-related factor 2): Cross-interaction between AhR (aryl hydrocarbon receptor) and Nrf2 signal transduction. Biochem J 377: 205-213, 2004.

39. He X, Chen MG, Lin GX and Ma Q: Arsenic induces NAD(P)Hquinone oxidoreductase I by disrupting the Nrf2 x Keap1 x.Cul3 complex and recruiting Nrf2 $x$.Maf to the antioxidant response element enhancer. J Biol Chem 281: 23620-23631, 2006.

40. Su JD, Yen JH, Li S, Weng CY, Lin MH, Ho CT and Wu MJ: 3',4'-didemethylnobiletin induces phase II detoxification gene expression and modulates PI3K/Akt signaling in PC12 cells. Free Radic Biol Med 52: 126-141, 2012. 